\title{
Editorial
}

\section{Variance, volatility swaps and hedging your equity portfolio}

Journal of Asset Management (2007) 8, 73. doi:10.1057/palgrave.jam.2250068

These are described as forward contracts on the sum of squared daily geometric rates of return relative to a reference value in the case of variance swaps, and a forward contract on the square root of the sum of squared daily geometric rates of return relative to a reference value in the case of a volatility swap. They hold out the promise, for an investor who has to hold a quantity of equity, of providing a hedge against market collapse. They are sold over the counter by a number of the large banks and a great deal of impressive research by clever people is extant proclaiming their robust properties.

Briefly, the analytic arguments in favour of variance swaps are based on the usual option pricing arguments, yet the strength of the claims that are made are surprisingly strong. It is claimed that valuation of a variance swap and hedging is model independent in a world where prices don't jump. It is further claimed that the volatility (vega) hedging portfolio is static and can be computed off the volatility smile in the same no-price jump world.

While musing over these claims, the following thoughts occur: pay-offs are nonlinear and we just know that in the discrete-time world we live in, hedging will always be approximate, thus the critical issue is whether the model works in a meltdown. To assess this we would need to go back to October 1987, strip out all the portfolio insurance that was then claimed to hedge equity, and see if variance swaps would have done better - I have not read that research yet but I do recall that portfolio insurance came in the same sort of package, wrapped in academic argument, rather widely accepted and eventually providing little relief for the insured.

Stephen E. Satchell Editor 